\title{
Psychometric evaluation of the sleep hygiene index: a sample of patients with chronic pain
}

\author{
Sungkun Cho', Gye-Seok Kim² and Jang-Han Lee ${ }^{3 *}$
}

\begin{abstract}
Background: Sleep Hygiene Index (SHI) was designed to assess sleep hygiene. Although the SHI has shown adequate psychometric properties in a nonclinical sample, it has not been validated in a sample with chronic pain. Also, its factor structure, measurement error, and incremental validity over and above other factors affecting sleep quality have not been investigated in a nonclinical sample. Thus, this present study aimed to extend prior psychometric investigation of the SHI. Specifically, we evaluated the factor structure, measurement error, and incremental validity as well as the reliabilities and concurrent validity of the SHI in a sample with chronic pain.

Methods: A total of 161 patients seeking treatment in a tertiary pain center located in Seoul, Korea participated. To explore the factor structure of the SHI, we performed an exploratory factor analysis using principal component with varimax. Cronbach's alphas and intraclass correlation coefficients were computed to investigate internal consistency and 2-week test-retest stability of the SHI, respectively. Measurement error was estimated using standard error of measurement and minimum detectable change (MDC) of the SHI. For concurrent validity, Pearson correlations were calculated to examine the relations between the SHI and outcome measures including background variables. Also for incremental validity, a hierarchical multiple regression was performed in relation to sleep quality.

Results: Results indicated that two-factor solution is most appropriate; sleep disturbing behavior and environment (B/E) and irregular sleep-wake schedule. Results also showed that the internal consistencies and test-retest stability estimates of the SHI were deemed acceptable. At the 95\% confidence level, the MDCs were 5.75 for 'sleep disturbing B/E,' 3.65 for 'irregular sleep-wake schedule,' and 7.49 points for total. The SHI was significantly correlated with age, depression, pain-related anxiety, and sleep quality. Also, sleep quality was significantly predicted by the irregular sleep-wake schedule subscale of the SHI, over and above background variables, pain intensity, depression, pain-related anxiety.
\end{abstract}

Conclusions: The SHI has the reliability, measurement error, and concurrent and incremental validity support for assessing sleep hygiene in a sample with chronic pain.

Keywords: Sleep Hygiene Index, Sleep quality, Chronic pain, Psychometric properties

\section{Background}

Poor sleep quality has been known as one of the most common complaints in patients with chronic pain (PCP) $[1,2]$. According to the National Sleep Foundation [3], approximately two-thirds of PCP suffer from poor sleep quality and their relative prevalence of poor sleep quality is four times that of the general population. Findings from earlier studies have indicated that poor sleep quality is linked to a wide range of deleterious outcomes,

\footnotetext{
* Correspondence: clipsy@cau.ac.kr

${ }^{3}$ Department of Psychology, Chung-Ang University, 221 Heukseok-dong, Dongjak-gu, Seoul 156-756, Korea

Full list of author information is available at the end of the article
}

such as greater pain, emotional distress, and physical and psychosocial disability [4-6]. In particular, persistent pain can impair sleep quality and impaired sleep quality can also lower pain tolerance, which can result in amplified sensations of pain. These reciprocal causal relations often lead PCP to fall into a vicious cycle [7-9]. Given these, it is reasonable to assume that effective pain management should enhance sleep quality to break the vicious cycle before it produces even worse problems.

Poor sleep quality in PCP has been reported to correlate with pain [10] and emotional distress $[10,11]$. Other than these factors, poor sleep quality in PCP may be also 
associated with behavioral and environmental factors such as poor sleep habits (referred to as sleep hygiene) [12]. Sleep hygiene refers to the daily practices, habits, and environmental factors that are necessary for improving the quality of a night-time sleep [13]. As pain becomes prolonged and persistent, PCP is more likely to develop poor sleep hygiene. For example, it is not uncommon that PCP stay inactive and rest in bed most of the day and exhibit irregular sleep-wake schedules [14]. Although the role of sleep hygiene on sleep quality has not been established in PCP, sleep hygiene is one of the first things to check when they complain about poor sleep quality [15].

There are, to our knowledge, three primary instruments designed to assess sleep hygiene: the Sleep Hygiene Awareness and Practice Scale (SHAPS) [16], the Sleep Hygiene Self-Test (SHST) [17], and the Sleep Hygiene Index (SHI) [18]. The first two instruments have been found to have relatively low internal consistency (Chronbach's alphas $=.47$ for the SHAPS and .54 for the SHST), compared to the SHI (Chronbach's alpha $=.66$ ). Moreover, these two instruments appeared to be developed with absence of clear rationale for item selection [18], while the SHI was developed from the diagnostic criteria for inadequate sleep hygiene as defined in the International Classification of Sleep Disorders [19]. The SHI has shown moderate internal consistency and good 2-week test-retest stability $(r=.71, p<.001)$ and been associated with sleep quality and daytime sleepiness in a nonclinical sample [18]. Although the SHI has shown adequate psychometric properties in a nonclinical sample, it has not been validated in a sample with chronic pain. Also, its factor structure, measurement error, and incremental validity over and above other factors affecting sleep quality have not been investigated in a nonclinical sample. Thus, this study aimed to 1) determine the factor structure of the $\mathrm{SHI}$ and 2) examine reliability, measurement error, and concurrent and incremental validity of the SHI over and above background variables, pain, and emotional distress (i.e., depression and pain-related anxiety) in PCP. As evidence for concurrent validity, poorer sleep hygiene is expected to significantly correlate with greater pain intensity, greater depression, greater pain-related anxiety, and lower sleep quality.

\section{Methods}

\section{Participants}

A total of 161 patients seeking treatment in a tertiary pain center located in Seoul, Korea participated. The sample was assessed at two time points, to examine test-retest stability over a 2-week interval of the SHI. After the patients completed an appointment at the pain center, volunteers were asked to complete the questionnaire packet in a private room. They also were provided with the identical questionnaire packet and a stamped addressed envelope for a second administration. They were instructed to take the packet to their home, complete and mail it back to the center after 2 weeks. One hundred thirteen patients (70\%) completed the packet at time 2 . Table 1 presents demographic characteristics of the sample. All data were collected and analyzed with approval by the Institutional Review Board (Seoul St. Mary's Hospital) and informed consent by participants.

\section{Translation}

The two Korean speaking clinical psychologists (one who specialized in chronic pain and was aware of the objective of the SHI; the other was not) separately translated the SHI into Korean. Any inconsistencies were adjusted based on the agreement between them. Then, a Korean-English bilingual graduate student who had no knowledge of the SHI back-translated it into English. The back-translation version was reviewed by one of the original authors of the SHI and revised accordingly. The pre-final version of the SHI was tested by 5 patients with chronic pain. Each patient was asked to complete the SHI and to provide feedback regarding the wording and meaning of the each item of the SHI. Thus, the pre-

\section{Table 1 Demographic characteristics of the sample}

\begin{tabular}{lc}
\hline Variable & Statistic \\
\hline Age (years) & \\
\hline SD & 44.9 \\
\hline Sex (\%) & 14.0 \\
\hline Men & 46.6 \\
\hline Women & 53.4 \\
\hline Marital status (\%) & \\
\hline Married & 66.5 \\
\hline Non-married & 33.5 \\
\hline Education level (\%) & \\
\hline$\geq$ High school & 90.4 \\
\hline Pain duration (months) & 36 \\
\hline Median & $3-480$ \\
\hline Range & 65.2 \\
\hline Taking pain-related medication (\%) & 39.6 \\
\hline Opioids & 60.4 \\
\hline Non-opioids & \\
\hline Most significant pain site(s) (\%) & 4.6 \\
\hline$\geq 2$ sites & 41.7 \\
\hline Lower back & 15.9 \\
\hline Leg(s) & \\
\hline Head & \\
\hline Others & \\
\hline
\end{tabular}


final version of the SHI was finally revised based on their feedback.

\section{Measures}

Demographic information was first obtained from a questionnaire. Items included age, sex, marital status, education level, and pain-related questions such as pain duration, pain-related medication, most significant pain site(s), and pain intensity. Pain intensity was measured by averaging present, usual, lowest, and highest pain during the past week, on a 0 (no pain) to 10 (worst possible pain) numeric rating scale (NRS). Total scores range from 0 to 10, with higher score representing worse pain. The NRS has been shown to have adequate reliability and validity in pain research [20].

The Sleep Hygiene Index (SHI) [18] is a 13-item selfreport measure designed to assess the practice of sleep hygiene behaviors. Each item is rated on a five-point scale ranging from 0 (never) to 4 (always). Total scores range from 0 to 52 , with a higher score representing poorer sleep hygiene. SHI has shown adequate reliability and validity [18].

The Center for Epidemiologic Studies Depressed Mood Scale (CES-D) [21] is a 20-item self-report measure designed to assess depressive symptoms. Each item is rated on a four-point scale ranging from 0 (rarely or none of the time) to 3 (most or all of the time). Total scores range from 0 to 60 , with a higher score representing greater depression. This study used a Korean language version of the CES-D which has been shown to have adequate reliability and validity [22].

The Pain Anxiety Symptoms Scale-20 (PASS-20) [23] is a 20-item self-report measure designed to assess painrelated anxiety. Each item is rated on a six-point scale ranging from 0 (never) to 5 (always). Total scores range from 0 to 100 , with a higher score representing greater pain-related anxiety. This study used a Korean version of the PASS-20 which has been shown to have adequate reliability and validity [24].

The Sleep Quality Scale (SQS) [25] is a 28-item selfreport measure designed to assess sleep quality. Each item is rated on a four-point scale ranging from 0 (rarely) to 3 (almost always). Total scores range from 0 to 84, with a higher score representing lower sleep quality. This study used a Korean version of the SQS which has been shown to have adequate reliability and validity [25].

\section{Statistical analysis}

The SPSS 18.0 for Windows software was used for the analyses. In order to explore the factor structure of the SHI, we performed an exploratory factor analysis (EFA) using principal component with varimax. Although rules of thumb regarding sample size for EFA have not yet been established, one common rule of thumb is a sample size of at least 100 [26] and another is a subject-to-item ratio of 10:1 [27]. Given this, the sample size $(\mathrm{N}=161)$ of the present study appeared to be minimally acceptable. The number of factors was retained based on eigenvalues, scree test, and parallel analysis using the mean eigenvalues and 95th percentile eigenvalues. Using eigenvalues greater than 1 has been considered to be the least accurate method for factor retention and thus, this study also employed alternative methods such as scree test and parallel analysis [28]. Also, only items with a factor loading of .32 or greater were retained. Tabachnick and Fidell [29] recommended .32 for a minimum factor loading, indicating that there is approximately $10 \%$ overlap in variance with the other items in that factor. An item loaded less than .32 or a cross-loading item was not retained. The final derived factor solution was then used for subsequent reliability and validity analyses. Cronbach's alphas and intraclass correlation coefficients $(\operatorname{ICC}(2,1))$ were computed to investigate internal consistency and 2-week test-retest stability of the SHI subscale and total scores, respectively. Measurement error was estimated using standard error of measurement (SEM) and minimum detectable change (MDC) of the SHI total and subscale scores. These were calculated by the following formulas: $\mathrm{SEM}=\mathrm{SD} \sqrt{ }(1-r)$, where $\mathrm{SD}$ is the baseline standard deviation of the measurement and $r$ is the 2-week testretest stability coefficient and MDC $=1.96^{*} \sqrt{ } 2^{*} \mathrm{SEM}$. For concurrent validity, Pearson correlations were calculated between the SHI and outcome measures including background variables. Dummy coding was used to represent a categorical variable (i.e., sex; male coded 0 , female coded 1). Given the number of correlations, a $p$ value was adjusted (i.e., $p<.006$ ) for more conservative prediction [30]. Also for the incremental validity of the SHI over and above background variables, pain intensity, depression, and pain-related anxiety, a hierarchical multiple regression was performed in relation to sleep quality (dependent variable). In each equation, participants' age, sex, education, and pain duration were controlled first, and pain intensity, depression, and pain-related anxiety were controlled next, followed by the two subscales of the SHI entered in the final step.

\section{Results}

EFA using principal component with varimax rotation was performed. The scree plot indicated that a 2 -factor model was optimal. Both factors had eigenvalues greater than 1. The parallel analysis also indicated a 2-factor model and thus, 2-factor model was retained. All of the items were saliently loaded on either factor, except items 4 and 8 . Specifically, item 4 was not saliently loaded on either factor and item 8 was saliently loaded on both factors. Thus, these items were excluded in the subsequent analyses. The 2 -factor model accounted for $40.01 \%$ of 
Table 2 Forced two-factor solution by principal component with varimax rotation of items from the SHI

\begin{tabular}{|c|c|c|c|c|c|c|c|}
\hline \multirow[b]{2}{*}{ Component } & \multicolumn{3}{|c|}{ Initial eigenvalues } & \multicolumn{3}{|c|}{ Extraction sums of squared loadings } & \multirow{2}{*}{$\frac{\text { Rotation sums of squared loading }}{\text { Total }}$} \\
\hline & Total & $\%$ of variance & Cumulative $\%$ & Total & $\%$ of variance & Cumulative \% & \\
\hline 1 & 3.33 & 25.59 & 25.59 & 3.33 & 25.59 & 25.59 & 2.65 \\
\hline 2 & 1.88 & 14.42 & 40.01 & 1.88 & 14.42 & 40.14 & 2.55 \\
\hline 3 & 1.19 & 9.15 & 49.17 & & & & \\
\hline 4 & 1.07 & 8.25 & 57.42 & & & & \\
\hline 5 & .99 & 7.60 & 65.02 & & & & \\
\hline 6 & .95 & 7.31 & 72.33 & & & & \\
\hline 7 & .78 & 6.01 & 78.34 & & & & \\
\hline 8 & .73 & 5.65 & 83.98 & & & & \\
\hline 9 & .59 & 4.50 & 88.48 & & & & \\
\hline 10 & .49 & 3.77 & 92.26 & & & & \\
\hline 11 & .44 & 3.36 & 95.62 & & & & \\
\hline 12 & .32 & 2.45 & 98.06 & & & & \\
\hline 13 & .25 & 1.94 & 100.00 & & & & \\
\hline
\end{tabular}

Note: SHI: Sleep Hygiene Index.

the total variance (43.23\% after excluding items 4 and 8 ) (Table 2), and the correlation coefficient among the factors was $.31, p<.001$ (.34 after excluding items 4 and 8 ), indicating a significant relationship among the factors. Considering the characteristics of the items loaded on each factor, factor 1 was labeled as 'sleep disturbing behavior and environment (B/E)' (e.g., I use alcohol, tobacco, or caffeine within 4 hours of going to bed or after going to bed) and factor 2 as irregular sleep-wake schedule' (e.g., I get out of bed at different times from day to day). Table 3 presents the factor loadings and item communalities of the 2 -factor model.

Table 4 presents descriptive statistics for the two subscale and total scores of the SHI and Table 5 presents the
Cronbach's $\alpha$, ICC $(2,1)$, SEM, and MDC for the sleep disturbing $\mathrm{B} / \mathrm{E}$ subscale, irregular sleep-wake schedule subscale, and total score. Table 6 presents descriptive statistics of the NRS, CES-D, PASS-20, and SQS. The data were all normally distributed except sex, education, and pain duration. However, given that Pearson's correlation does not assume normality [31] and a non-parametric test (Spearman rank correlation) showed the similar results, we evaluated the relationship among all of the variables using Pearson's $r$. Table 7 presents Pearson correlations between the SHI and outcome measures including background variables. Findings for background variables were mixed. Age was significantly negatively correlated with both subscales and total scores of the SHI, indicating younger individuals

Table 3 Two-factor solution: Factor loadings and communalities by principal components from the SHI

\begin{tabular}{|c|c|c|c|}
\hline \multirow[b]{2}{*}{ Item content } & \multicolumn{2}{|c|}{ Factor loadings } & \multirow[b]{2}{*}{$h^{2}$} \\
\hline & 1 & 2 & \\
\hline 10. I sleep on an uncomfortable bed (for example: poor mattress or pillow, too much or not enough blankets). & .77 & .06 & .60 \\
\hline 11. I sleep in an uncomfortable bedroom (for example: too bright, too stuffy, too hot, too cold, or too noisy). & .74 & -.02 & .55 \\
\hline 12. I do important work before bedtime (for example: pay bills, schedule, or study). & .65 & -.12 & .43 \\
\hline 7. I do something that may wake me up before bedtime (for example: play video games, use the internet, or clean). & .51 & .23 & .31 \\
\hline 6. I use alcohol, tobacco, or caffeine within 4 hours of going to bed or after going to bed. & .50 & .04 & .25 \\
\hline 13. I think, plan, or worry when I am in bed. & .49 & .20 & .28 \\
\hline 9. I use my bed for things other than sleeping or sex (for example, watch television, read, eat, or study). & .43 & .26 & .25 \\
\hline 4. I exercise to the point of sweating within 1 hour of going to bed. & .14 & .06 & .02 \\
\hline 3. I get out of bed at different times from day to day. & .13 & .83 & .70 \\
\hline 2. I go to bed at different times from day to day. & .13 & .82 & .69 \\
\hline 5. I stay in bed longer than I should two or three times a week. & .06 & .74 & .55 \\
\hline 1. I take daytime naps lasting two or more hours. & .00 & .50 & .25 \\
\hline 8. I go to bed feeling stressed, angry, upset, or nervous. & .34 & .46 & .33 \\
\hline
\end{tabular}

Note: Bold number indicates salient factor loading $(\geq .32) ; h^{2}$ indicates item communalities; SHI: Sleep Hygiene Index. 
Table 4 Descriptive statistics for subscale and total scores of the SHI

\begin{tabular}{lccccc}
\hline Subscale & \# items & $\begin{array}{c}\text { Possible } \\
\text { range }\end{array}$ & M & SD & Intercorrelations \\
\hline 1. Sleep disturbing B/E & 7 & $0-28$ & 11.45 & 4.33 & $\mathbf{1}$ \\
\hline 2. Irregular sleep-wake schedule & 4 & $0-16$ & 7.70 & 3.21 & $.34^{* * *}$ \\
\hline 3. Total & 11 & $0-44$ & 19.16 & 6.58 & $.85^{* * *}$ \\
\hline
\end{tabular}

Note: ${ }^{* * *} p<.001$. SHI: Sleep Hygiene Index; B/E: behavior and environment.

reported poorer sleep hygiene. Neither the subscale nor total scores of the SHI were significantly correlated with sex, education level, and pain duration. Findings for the four outcomes were also mixed, partly supporting the hypotheses. Greater depression and pain-related anxiety were significantly positively correlated with more irregular sleep-wake schedule and total score, but not sleep disturbing $\mathrm{B} / \mathrm{E}$. Sleep quality was significantly positively correlated with both subscales and total score of the SHI. Neither the subscale nor total scores of the SHI were significantly correlated with pain intensity. Incremental validity of the SHI over and above background variables, pain intensity, depression, and pain-related anxiety was further examined using hierarchical multiple regressions of the two subscales of the SHI in relation to sleep quality (dependent variable). The assumptions of multiple linear regression (i.e., linearity, independence of errors, homoscedasticity, multicollinearity, normality of errors) were satisfied. Generally, the finding indicated significant overall relation in the equation. Two subscales of the SHI added a significant increment in explained variance in the equation $\left(\Delta \mathrm{R}^{2}=.02, p<.05\right)$. In the final step, the regression coefficients for irregular sleepwake schedule $(\beta=-.67, p<.05)$, but not for sleep disturbing $\mathrm{B} / \mathrm{E}(\beta=.17$, n.s. $)$ were significant in the equation (Table 8).

\section{Discussion}

This present study aimed to extend a prior psychometric investigation of the SHI which assessed the reliabilities and concurrent validity in a nonclinical sample [18]. Specifically, we evaluated the factor structure, measurement error, and incremental validity as well as the reliabilities and concurrent validity of the SHI in a sample with chronic pain. Results from the present study indicated that two-factor solution is most appropriate, accounting for $43.23 \%$ of the variance. We interpreted the two factors as indicative of sleep disturbing $\mathrm{B} / \mathrm{E}$ (7 items) and irregular sleep-wake schedule (4 items). Results also showed that the internal consistencies and test-retest stability estimates of the SHI subscale and total scores were deemed acceptable and relatively high compared to the prior study [18]. At the 95\% confidence level, the MDCs were 5.75 for 'sleep disturbing B/E', 3.65 for 'irregular sleep-wake schedule', and 7.49 points for total. Changes over and above these points after intervention would likely not be measurement error.

Correlation analyses showed that age is negatively correlated with both subscales and total scores of the SHI. Overall, these findings suggest that younger people tend to show worse sleep hygiene. Depression and pain-related anxiety were positively correlated with the irregular sleepwake schedule subscale and total scores of the SHI, suggesting that greater depression and pain-related anxiety are associated with worse sleep hygiene (especially regarding the aspect of irregular sleep-wake schedule). Both subscales and total scores of the SHI were positively correlated with sleep quality, suggesting that poorer sleep hygiene is associated with poorer sleep quality, which is consistent with prior studies [18]. Neither the subscale nor total scores of the SHI were significantly correlated with pain intensity. These findings suggest that pain may not be directly related to sleep hygiene. Instead, given that pain has been often considered to produce depression and pain-related anxiety [34], pain may be indirectly related to sleep hygiene. Further studies may benefit from examining possible interactions between pain intensity and depression and/or pain-related anxiety, in their relations with sleep hygiene.

In terms of incremental validity in relation to sleep quality, two subscales of the SHI added a significant increment in explained variance in the equation. Also, sleep quality was significantly predicted by the irregular sleep-wake schedule subscale only, over and above background variables and some primary factors affecting

Table 5 Internal consistency, 2-week test-retest reliability, and measurement error of the SHI

\begin{tabular}{lcccc}
\hline Subscale & Chronbach's a & ICC (2, 1) (95\% Cl) & SEM & MDC \\
\hline Sleep disturbing B/E & .74 & $.77(.73-89)$ & 2.08 & \\
\hline Irregular sleep-wake schedule & .70 & $.83(.76-.88)$ & 1.32 & \\
\hline Total & .75 & $.83(.73-.89)$ & 2.75 \\
\hline
\end{tabular}

Note: ICC: intraclass correlation coefficient; SEM: standard error of measurement; MDC: minimum detectable change; B/E: behavior and environment. 
Table 6 Descriptive statistics for the NRS, CES-D, PASS-20, and SQS

\begin{tabular}{lccc}
\hline & Possible range & M & SD \\
\hline NRS (pain intensity) & $0-10$ & 5.31 & 2.31 \\
\hline CES-D & $0-60$ & 26.91 & 13.46 \\
\hline PASS-20 & $0-100$ & 46.74 & 21.50 \\
\hline SQS & $0-84$ & 66.86 & 18.57 \\
\hline
\end{tabular}

Note: NRS: Numerical Rating Scale; CES-D: Center for Epidemiologic Studies Depression Scale; PASS-20: Pain Anxiety Symptom Scale-20; SQS: Sleep Quality Scale.

sleep quality (i.e., pain intensity, depression, pain-related anxiety) in PCP. This finding suggests the unique contribution of sleep hygiene to sleep quality and provides evidence for relative importance of sleep-wake schedule over sleep disturbing $\mathrm{B} / \mathrm{E}$ in sleep quality of $\mathrm{PCP}$. In fact, many PCP develop an irregular sleep-wake pattern through pharmacological and/or behavioral factors [35]. For example, PCP often take multiple medications and some of these (e.g., opioid and benzodiazepines) change their sleep-wake schedule. Also, many PCP fail to maintain a consistent daily activity pattern and spend considerable time in bed or inactive. More specifically, they may be in bed or inactive during episodes of severe pain. On the other hand, in response to some pain relief, they may overdo things that they were unable to do during the episodes of severe pain. This often leads to increases in pain followed by a few days of bed rest or inactivity for recovery. As a result, their sleep-wake schedule can be impaired, leading to poor sleep quality [35].

This present study has at least four limitations. First, although the process of translation/backtranslation was performed, it did not fully meet the guidelines for the process of cross-cultural adaptation of self-report measures [36].

Table 7 Correlations between the SHI subscale and total scores and background and outcome variables

\begin{tabular}{lccc}
\hline & $\begin{array}{c}\text { Sleep } \\
\text { disturbing B/E }\end{array}$ & $\begin{array}{c}\text { Irregular sleep-wake } \\
\text { schedule }\end{array}$ & $\begin{array}{c}\text { Total } \\
\text { score }\end{array}$ \\
\hline Age & -.26 & $-.38^{*}$ & $-.39^{*}$ \\
\hline Sex ${ }^{\text {a }}$ & -.23 & -.14 & -.24 \\
\hline Education & .05 & -.05 & .01 \\
\hline Pain duration & -.08 & -.03 & -.07 \\
\hline Pain intensity & -.03 & .15 & .06 \\
\hline Depression & .12 & $.51^{*}$ & $.37^{*}$ \\
\hline Pain-related & .15 & $.46^{*}$ & $.35^{*}$ \\
anxiety & & & $.41^{*}$ \\
\hline Sleep quality & .17 & $.52^{*}$ & $1 . \mathrm{SH}^{*}$
\end{tabular}

Note: ${ }^{*} p<.006$ adjusted $p$ level of 0.006 . ${ }^{a}$ male coded 0 , female coded 1 . SHI: Sleep Hygiene Index; B/E: behavior and environment. For concurrent validity, it was hypothesized that the $\mathrm{SHI}$ subscale and total scores are significantly positively correlated with four outcome variables (i.e., pain intensity, depression, pain-related anxiety, sleep quality).
Table 8 Results of hierarchical regression analyses predicting sleep quality from the two subscales of the SHI

\begin{tabular}{|c|c|c|c|c|}
\hline Step & Predictor & $\beta$ (Final) & $\Delta \mathrm{R}^{2}$ & Total $\mathrm{R}^{2}$ \\
\hline \multirow[t]{4}{*}{1.} & Age & -.01 & & \\
\hline & Sex & .06 & & \\
\hline & Education & -1.15 & & \\
\hline & Pain duration & .00 & $.07^{*}$ & \\
\hline \multirow[t]{3}{*}{2.} & Pain intensity & .14 & & \\
\hline & Depression & $.83^{* * * *}$ & & \\
\hline & Pain-related anxiety & .07 & $.55^{* * *}$ & \\
\hline \multirow[t]{2}{*}{3.} & Sleep disturbing $\mathrm{B} / \mathrm{E}$ & .17 & & \\
\hline & Irregular sleep-wake schedule & $-.67^{*}$ & $.02^{*}$ & $.63^{* * *}$ \\
\hline
\end{tabular}

Note: ${ }^{*} p<.05,{ }^{* * *} p<.001$. SHI: Sleep Hygiene Index; B/E: behavior and environment. Sex, education, and pain duration were not normally distributed. Thus, normality was further checked through histograms of the standardized residuals [32], and Q-plots and P-plots [33]. These tests supported the assumption of normality.

Thus, items of the translated SHI may not be identical in meaning with those of the original SHI. Insufficient equivalence in meaning is more likely to limit the comparability of responses across different languages or cultures [36]. Second, although the sample size of this present study appeared to be minimally acceptable for EFA, a larger sample can help obtain a more stable solution [37] and increase generalizability to the population [38]. Third, this present study did not evaluate responsiveness of the SHI and cannot provide the criterion for clinically relevant change. Thus, responsiveness of the SHI scores to interventions should be evaluated in further research for the use of the SHI as a sign of changes in PCP. Finally, this present study employed a cross-sectional design and was composed of patients with diverse pain complaints, attending a tertiary pain center. Thus, causal relations among variables cannot be inferred and the findings may not generalize to other pain population (e.g., fibromyalgia, complex regional pain syndrome) or other settings (e.g., primary or secondary care clinic).

\section{Conclusions}

The findings of this present study suggest that the SHI can be a useful research or clinical assessment tool for evaluating sleep hygiene to guide case formulation, treatment planning, or process analysis of intervention in pain centers. This present study provided evidence for the factor structure, reliability, measurement error, and concurrent and incremental validity of the SHI in PCP. However, given that this present study was the first attempt to validate the SHI in a sample with chronic pain, its application in clinical settings should come after further studies, including its utility in clinical decision support and aid in developing further treatment. 


\section{Competing interests}

The authors declare that they have no competing interests.

\section{Authors' contributions}

All of the authors have contributed in this study with design, data collection, and interpretation of the results. The manuscript was drafted by SC with supervision of GSK and JHL. All authors have read and corrected draft versions, and approved the final version.

\section{Acknowledgement}

This research was supported by Basic Science Research Program through the National Research Foundation of Korea (NRF) funded by the Ministry of Education, Science and Technology (2012R1A1A2008624).

\section{Author details}

${ }^{1}$ Department of Psychology, Chungnam National University, 99 Daehak-ro, Yuseong-gu, Daejeon 305-764, Korea. ${ }^{2}$ Department of Business Administration, Sangji University, 660 Usan-dong Wonju-si, Gangwon-do, Korea. ${ }^{3}$ Department of Psychology, Chung-Ang University, 221 Heukseok-dong, Dongjak-gu, Seoul 156-756, Korea.

Received: 17 May 2013 Accepted: 17 December 2013

Published: 22 December 2013

\section{References}

1. Hurley DA, Eadie J, O'Donoghue G, Kelly C, Lonsdale C: Physiotherapy for sleep disturbance in chronic low back pain: a feasibility randomised controlled trial. BMC Musculoskel Dis 2010, 11:70.

2. Menefee LA, Cohen MJ, Anderson WR, Doghramiji K, Frank ED, Lee H: Sleep disturbance and nonmalignant chronic pain: a comprehensive review of literature. Pain Med 2000, 1:156-172.

3. National Sleep Foundation: Ask the sleep expert: pain and sleep. http://www.sleepfoundation.org/article/ask-the-expert/pain-and-sleep.

4. McCracken LM, Iverson GL: Disrupted sleep patterns and daily functioning in patients with chronic pain. Pain Res Manag 2002, 7:75-79.

5. Moldofsky H: Sleep and pain. Sleep Med Rev 2001, 5:385-396.

6. Smith MT, Haythornthwaite JA: How do sleep disturbance and chronic pain inter-relate? Insights from the longitudinal and cognitive behavioral clinical trials literature. Sleep Med Rev 2004, 8:119-132.

7. Drewes AM: Pain and sleep disturbances with special references to fibromyalgia and rheumatoid arthritis. Rheumatology 1999, 38:1035-1038.

8. Drewes AM, Arendt-Nielsen L: Pain and sleep in medical diseases: interactions and treatment possibilities. Sleep Res Online 2001, 4:67-76.

9. Kundermann B, Krieg JC, Schreiber W, Lautenbacher S: The effect of sleep deprivation on pain. Pain Res Manag 2004, 9:25-32.

10. Pilowsky I, Crettenden I, Townley M: Sleep disturbance in pain clinic patients. Pain 1985, 23:27-33.

11. Wilson KG, Watson ST, Currie SR: Daily and ambulatory activity monitoring of sleep in patients with insomnia associated with chronic musculoskeletal pain. Pain 1998, 75:75-84.

12. Currie SR, Wilson KG, Pontefract AJ, deLaplante L: Cognitive-behavioral treatment of insomnia secondary to chronic pain. J Consult Clin Psychol 2000, 68:407-416.

13. Riedel BW: Sleep hygiene. In Treatment of of late-life insomnia. Edited by Lichstein KL, Morin CM. Thousand Oaks, CA: Sage; 2000.

14. Deardorff WW: Practicing good sleep hygiene. http://www.spine-health. com/wellness/sleep/practicing-good-sleep-hygiene.

15. Perlis LM: Cognitive behavioral treatment of insomnia. New York, NY: Springer; 2005.

16. Lacks $P$, Rotert M: Knowledge and practice of sleep hygiene techniques in insomniacs and good sleepers. Behav Res Ther 1986, 24:365-368.

17. Blake DD, Gomez MH: A scale for assessing sleep hygiene: Preliminary data. Psychol Rep 1998, 83:1175-1178.

18. Mastin DF, Bryson J, Corwyn R: Assessment of sleep hygiene using the sleep hygiene index. J Behav Med 2006, 29:223-227.

19. American Sleep Disorders Association: International Classification of Sleep Disorders: Diagnostic and Coding Manual. Rochester: MN: American Sleep Disorders Association; 1990.

20. Jensen MP, Karoly P: Self-report scales and procedures for assessing pain in adults. In Handbook of pain assessment. Edited by Turk DC, Melzack R. New York, NY: Guilford; 2001.
21. Radloff LS, The CES-D scale: A self-report depression scale for research in the general population. Appl Psych Meas 1977, 1:385-401.

22. Chon KK, Rhee MK: Preliminary development of Korean version of CES-D. Kor J Clin Psychol 1992, 11:65-76.

23. McCracken $L M$, Dhingra $L$ : A short version of the Pain Anxiety Symptoms Scale (PASS-20): Preliminary development and validity. Pain Res Manag 2002, 7:45-50.

24. Cho S, Lee SM, McCracken LM, Moon DE, Heiby EM: Psychometric properties of a Korean Version of the Pain Anxiety Symptoms Scale-20 in chronic pain patients. Int J Behav Med 2010, 17:108-117.

25. Yi H, Shin K, Kim J, Kim J, Lee JB, Shin C: Validity and reliability of Sleep Quality Scale in subjects with obstructive sleep apnea syndrome. J Psychosom Res 2009, 66:85-88.

26. Hair J, Anderson RE, Tatham RL, Black WC: Multivariate data analysis. 4th edition. New Jersey: Prentice-Hall Inc; 1995.

27. Cabrera-Nguyen P: Author guidelines for reporting scale development and validation results in the Journal of the Society for Social Work and Research. J Soc Work Res 2010, 1:99-103.

28. Velicer WF, Fava JL: Effects of variable and subject sampling on factor pattern recovery. Psychol Methods 1998, 3:231-251.

29. Tabachnick BG, Fidell LS: Using Multivariate Statistics. Boston: Allyn and Bacon; 2001

30. Cano A: Pain catastrophizing and social support in married individuals with chronic pain: the moderating role of pain duration. Pain 2004, 110:656-664.

31. Rodgers $\lrcorner$, Nicewander WA: Thirteen ways to look at the correlation coefficient. Am Stat 1988, 42:59-66.

32. Stevens JP: Applied multivariate statistics for the social sciences. 5th edition. New York, NY: Routledge; 2009.

33. Keith T: Multiple regression and beyond. Boston, MA: Allyn \& Bacon; 2006.

34. Fisher BJ, Cutler R, Rosomoff HL, Rosomoff RS: Chronic pain associated with depression: Antecedent or consequence of chronic pain? A review. Clin J Pain 1997, 13:116-137.

35. Cohen MJM, Menefee LA, Doghramji K, Anderson WR, Frank ED: Sleep in chronic pain: problems and treatments. Int Rev Psychiat 2000, 12:115-126.

36. Beaton D, Bombardier C, Guillemin F, Ferraz M: Guidelines for the process of cross-cultural adaptation of self-report measures. Spine 2000, 25:3186-3191.

37. Cliff N: The relation between sample and population characteristic vectors. Psychometrika 1970, 35:163-178.

38. MacCallum RC, Widaman KF, Zhang S, Hong S: Sample size in factor analysis. Psychol Methods 1999, 4:84-99.

doi:10.1186/1477-7525-11-213

Cite this article as: Cho et al.: Psychometric evaluation of the sleep hygiene index: a sample of patients with chronic pain. Health and Quality of Life Outcomes 2013 11:213.

\section{Submit your next manuscript to BioMed Central and take full advantage of:}

- Convenient online submission

- Thorough peer review

- No space constraints or color figure charges

- Immediate publication on acceptance

- Inclusion in PubMed, CAS, Scopus and Google Scholar

- Research which is freely available for redistribution 